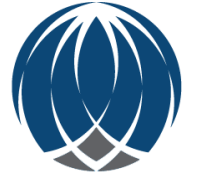

\section{A L LEN}

BRAIN ATLAS

MOUSE BRAIN

\title{
ANTERIOR OLFACTORY NUCLEUS (AON)
}

\author{
Beth M. Solan, Lydia L. Ng and Angela L. Guillozet-Bongaarts
}

\section{Introduction}

This report contains a gene expression summary of the anterior olfactory nucleus (AON), derived from the Allen Brain Atlas (ABA) in situ hybridization mouse data set. The structure's location and morphological characteristics in the mouse brain are described using the Nissl data found in the Allen Reference Atlas. Using an established algorithm, the expression values of the AON were compared to the values of the macro/parent-structure, in this case the olfactory areas, for the purpose of extracting regionally selective gene expression data. The genes with the highest ranking selectivity ratios were manually curated and verified. 50 genes were then selected and compiled for expression characterization. The experimental data for each gene may be accessed via the links provided; additional data in the sagittal plane may also be accessed using the $A B A$. Correlations between gene expression in the AON and the rest of the brain, across all genes in the coronal dataset ( 4300 genes), were derived computationally. A gene ontology table (derived from DAVID Bioinformatics Resources 2007) is also included, highlighting possible functions of the 50 genes selected for this report.

To read more about how our 50 Select Genes list is derived, please refer to the Fine Structure Annotation white paper.

Allen Reference Atlas Coronal Levels: 19-38

Allen Reference Atlas Sagittal Levels: 11-19

Shown below is a plate from the Allen Reference Atlas, depicting the AON (level $\underline{28}$ ):

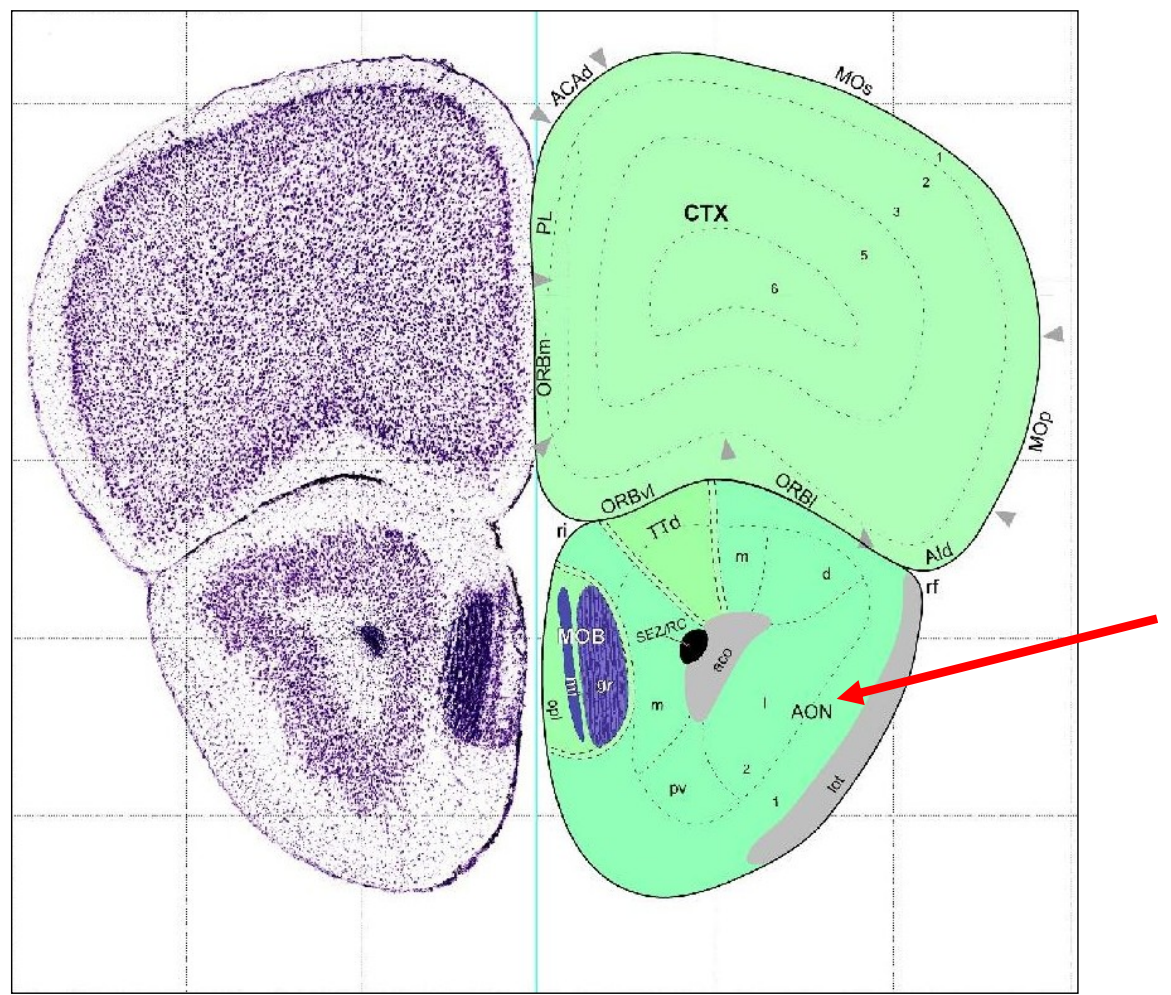




\section{LOCATION and STRUCTURAL ANATOMY:}

The hierarchical relationship within the brain is depicted below in the structure legend. The Allen Reference Atlas (based on Nisslstained sections scanned at 10X) was the primary resource for the following descriptions.

For additional information please refer to the Allen Reference Atlas white paper. BrainInfo houses a search engine that allows searches for structure name aliases.

The anterior olfactory nucleus (AON) is a substructure of the olfactory areas, located within the rostral portion of the cerebral cortex. The AON is comprised of dorsal (AONd), external $(A O N e)$, lateral (AONI), medial (AONm) and posteroventral (AONpv) subdivisions. Rostrally, the AONe surrounds the remaining subdivisions, and is therefore easily distinguished from them; the borders between the internal nuclei are difficult to distinguish.

In the coronal plane, the AON presents most rostrally within the main olfactory bulb (MOB). It can first be seen at the lateral aspect of the MOB bordering the subependymal zone/rhinocele (SEZI $\mathrm{RC}$ ). Caudally, the AONe disappears and the internal areas spread medially until they fully surround the SEZ/RC. At its most caudal aspect, the AONI is no longer present, and only the AONm and $A O N p v$ subdivisions remain. The caudal edge of the $A O N$ is consistent with the emergence of the nucleus accumbens.

In sagittal sections, the AON emerges just lateral to the junction of the MOB and the rest of the brain. The AON lies caudal to the MOB and rostro-dorsal to the piriform cortex. Continuing medially, the AON appears on either side of the SEZ/RC and the olfactory limb of the anterior commissure, and extends slightly rostrally into the MOB. At the midline, the AON is supplanted by the taenia tecta (TT).

The cells in the AONe are more densely packed than in the internal areas. The internal nuclei are separated from the AONe by a thin, cell-sparse layer. The size of the cells in the AON make this structure easily distinguished from the anterior commissure and the lateral olfactory tract.

The appearance and location of the AON can be appreciated on the following two pages. Nisslstained sections and Allen Reference Atlas plates reveal the cytoarchitecture and extent of the $A O N$, and its location in relation to surrounding structures.

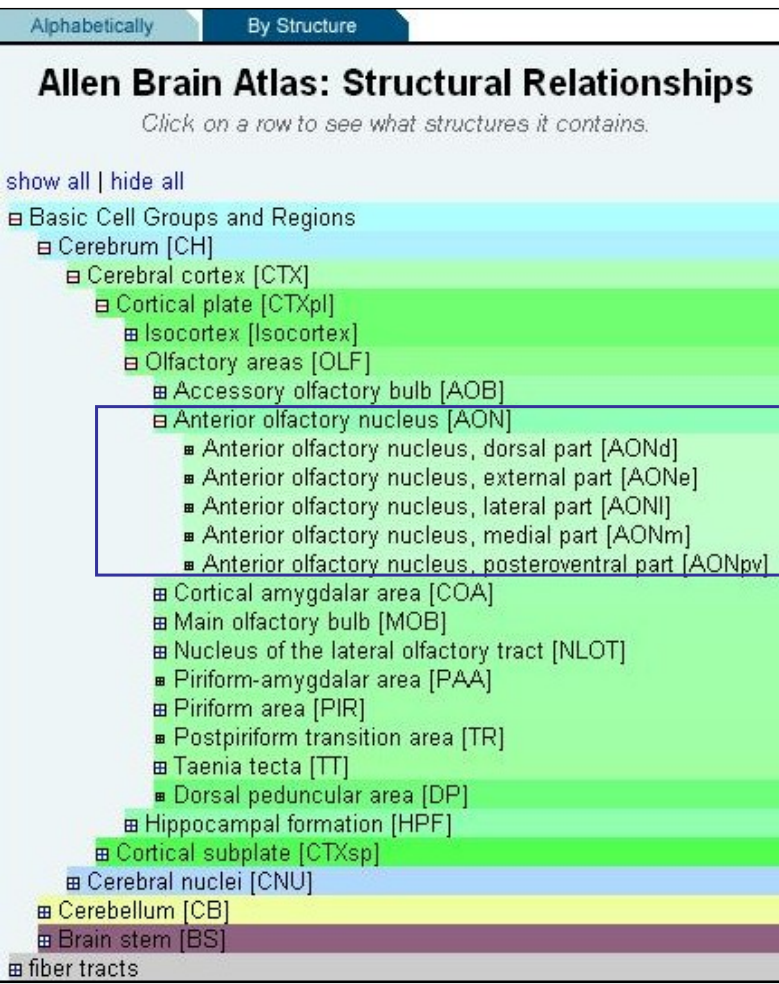




\section{Atlas and Nissl: Coronal:}

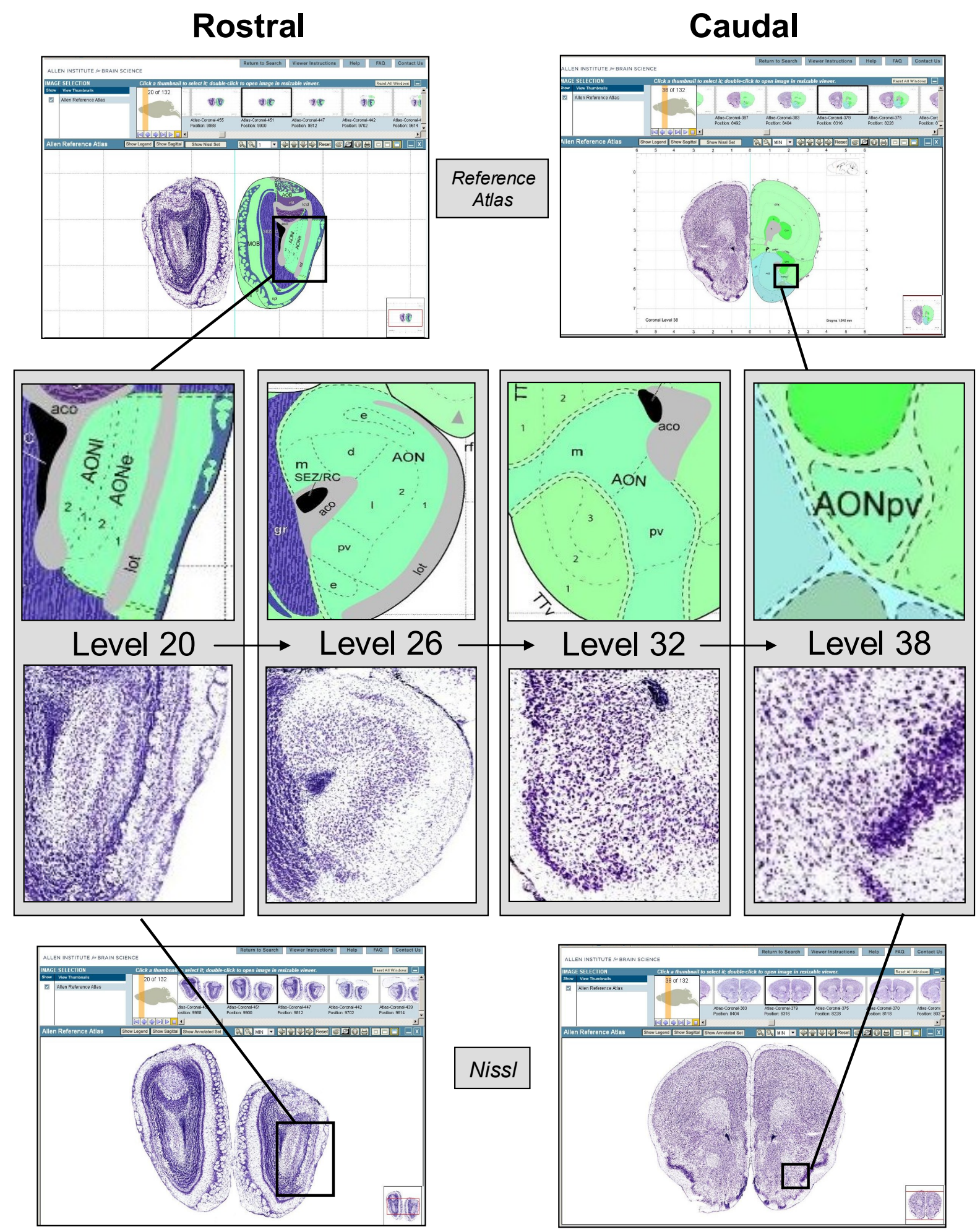




\section{Atlas and Nissl: Sagittal:}

Lateral

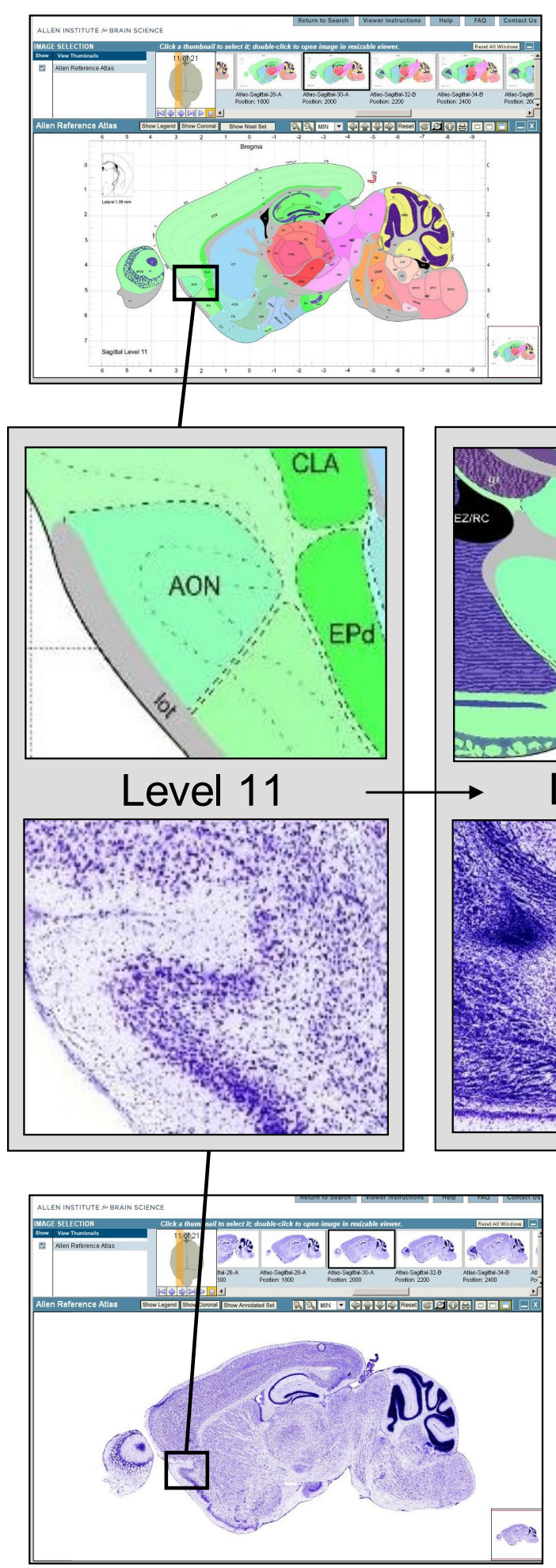

Medial

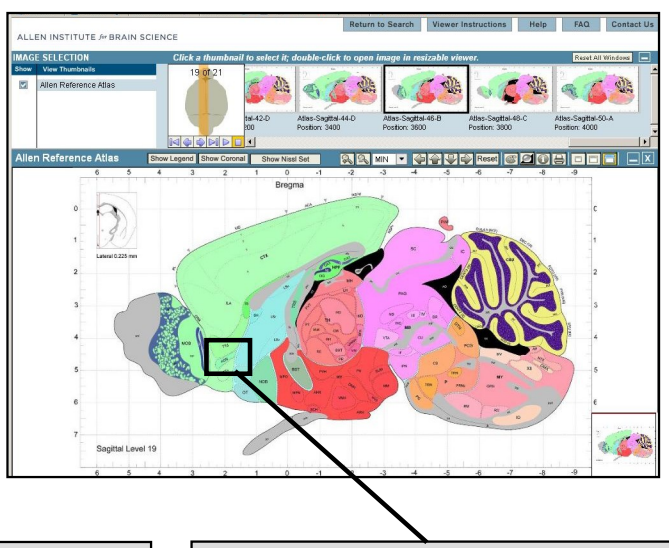

Reference Atlas
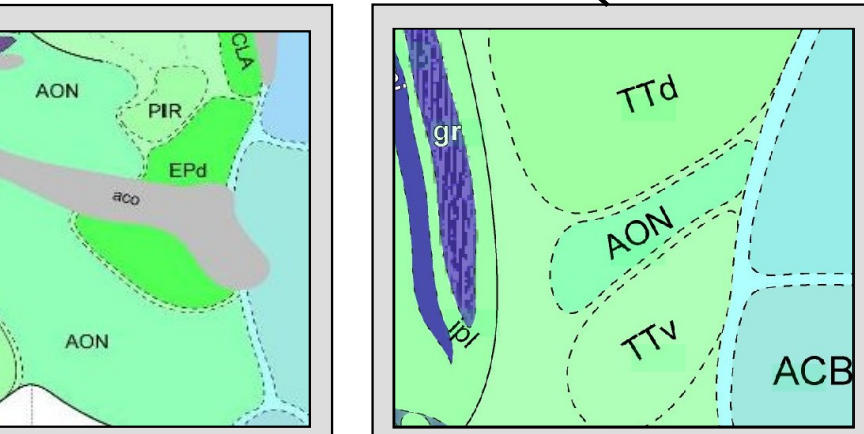

Level 15
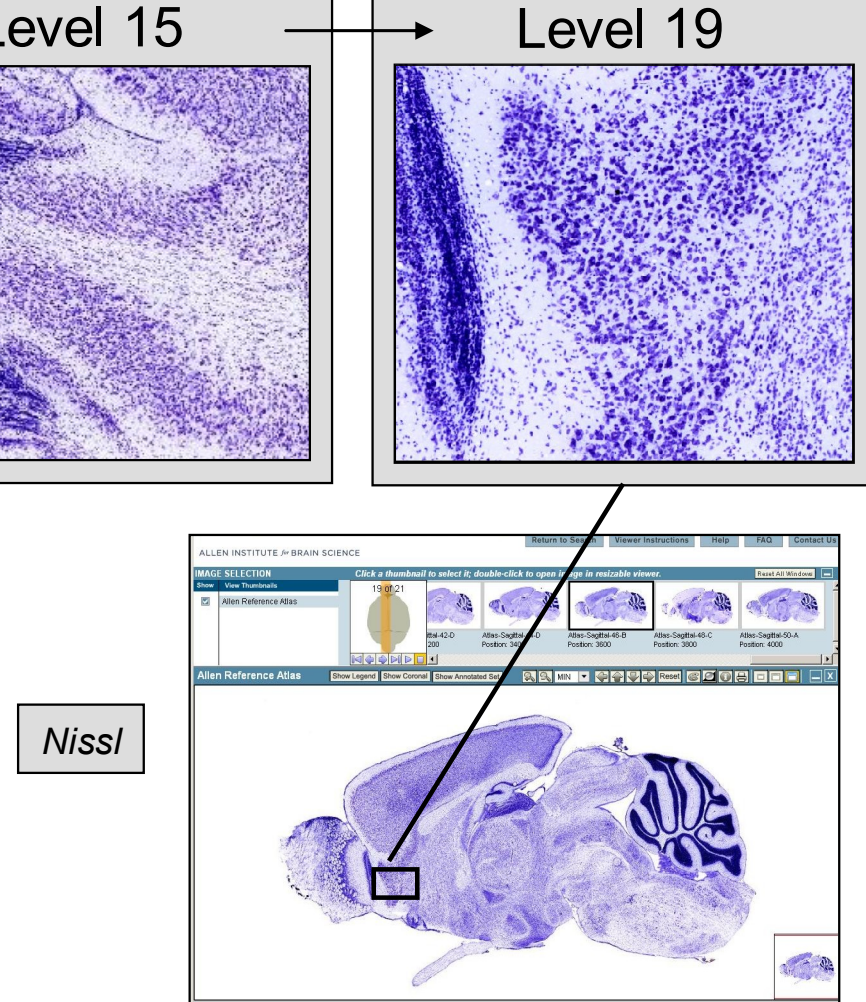
The in situ hybridization (ISH) data below presents the anterior olfactory nucleus' anatomical and cytoarchitectural characteristics in the context of actual gene expression. In addition to presenting molecularly defined borders, ISH gene expression patterns also aid in phenotyping cell populations that otherwise can not be differentiated on purely morphological grounds.

The 50 genes in this section were selected based on a mathematical algorithm to identify gene expression patterns that allow selective identification of the anterior olfactory nucleus. The gene expression patterns were then verified manually. As such, these genes do not represent the only genes found in this structure, genes specific to this structure, or genes expressing at the highest level within this structure.

Please refer to our protocol in the Data Production Processes white paper.

To read about heat map conversion, refer to the Informatics Data Processing white paper.

The expression data subsequently presented can be further explored, in coronal and sagittal planes, at brain-map.org.

Two major portions of the AON can be distinguished based on gene expression: the AONe and the remaining internal areas ( $\mathrm{AONI}, \mathrm{AONm}$, and $\mathrm{AONpv}$ ). This set of genes display three expression patterns. Approximately half of the genes presented are expressed throughout the entire AON, with similar expression in the internal and external regions. The remaining genes are either preferentially expressed in the AONe or are preferentially expressed in the internal areas. The AON is easily distinguished from its surrounding structures in these ISH studies, although the individual nuclei of the internal portions remain equally difficult to distinguish.

\begin{tabular}{|l|l|l|l|}
\hline \multicolumn{2}{|l|}{ Cellular density expression key } & \multicolumn{2}{l|}{ Cellular intensity expression key } \\
\hline None & No expression & No color & Very low intensity \\
\hline Sparse & Very few cells expressing & Blue & Low intensity \\
\hline Scattered & Less than $10 \%$ of cells expressing in scattered pattern & Green & Medium intensity \\
\hline Medium & $10-80 \%$ of cells expressing & Yellow & High intensity \\
\hline High & Greater than $80 \%$ of cells expressing & Red & Very high intensity \\
\hline
\end{tabular}

To view heat map at brain-map.org, right click on the ISH image and select "Show Expression Analysis."

ISH DATA The images below were selected to highlight various expression patterns of the AON.

\section{ISH}

Mpped1

Coronal: The gene expressed below allows the visualization of the external nucleus and the internal areas of the AON.

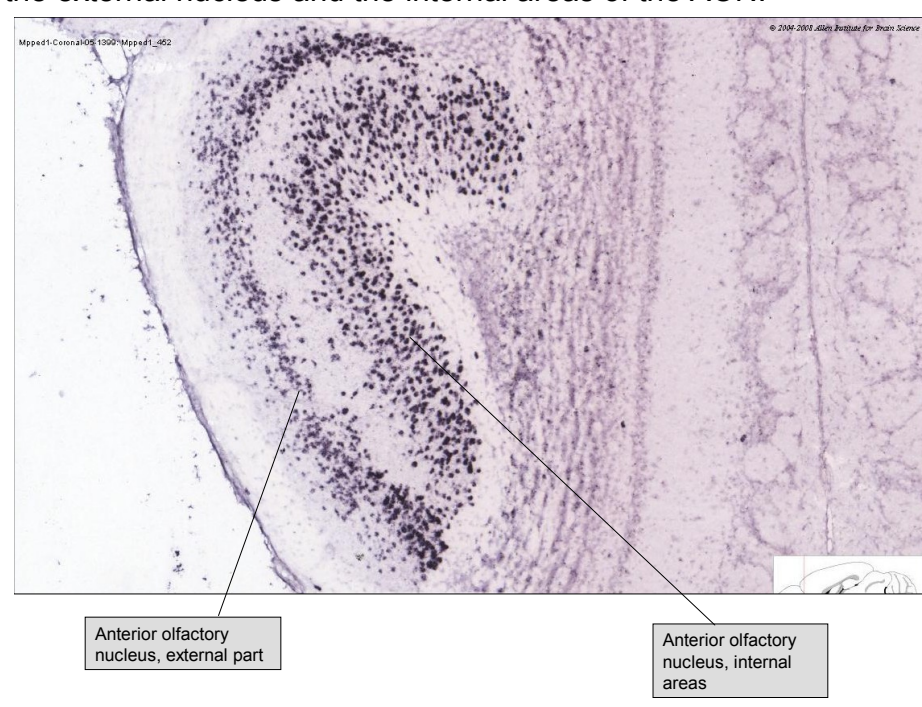

\section{Heat map}

Mpped1

Coronal: Both the external and internal parts of the AON show high density and intensity expression for gene Mpped 1.

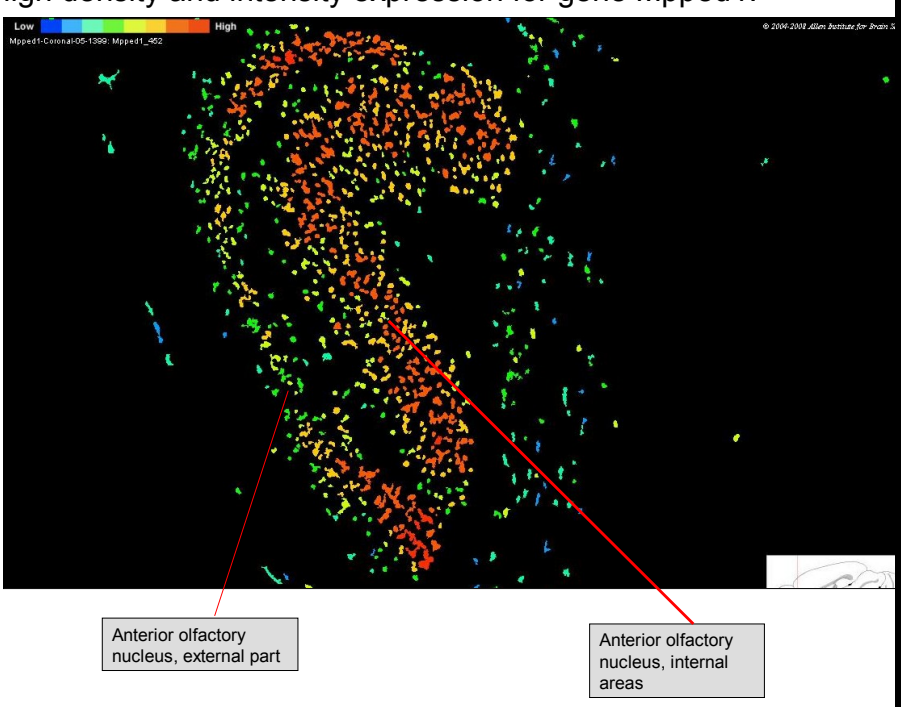




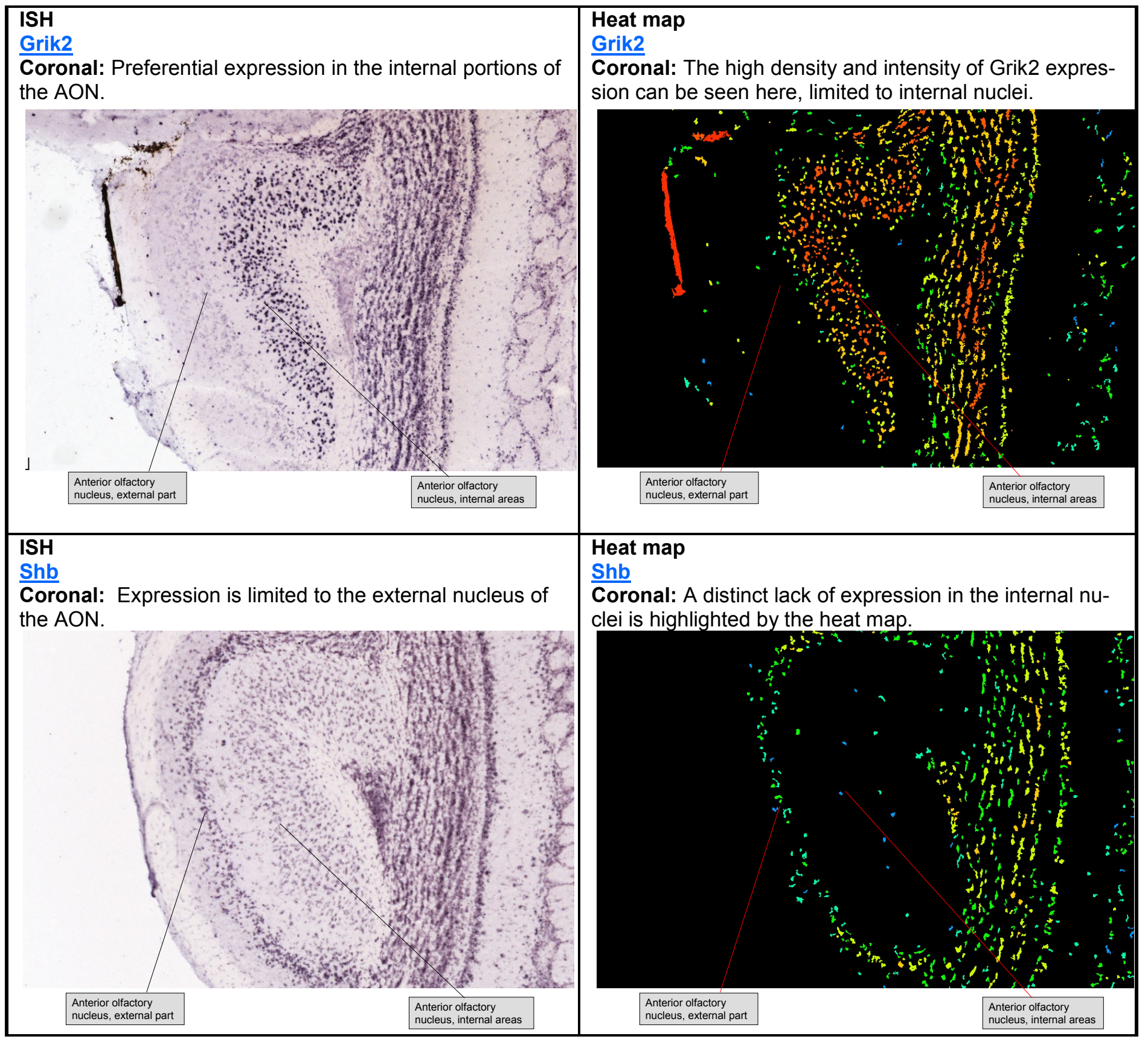


50 SELECT GENES:

This gene list was generated by manual curation of an algorithmically derived list that compared gene expression values of the AON to those of the olfactory areas. Categories of expression are subjectively grouped by relative expression characteristics.

Curation of 50 Select Genes List: February 2008

\section{Widespread expression throughout the AON}

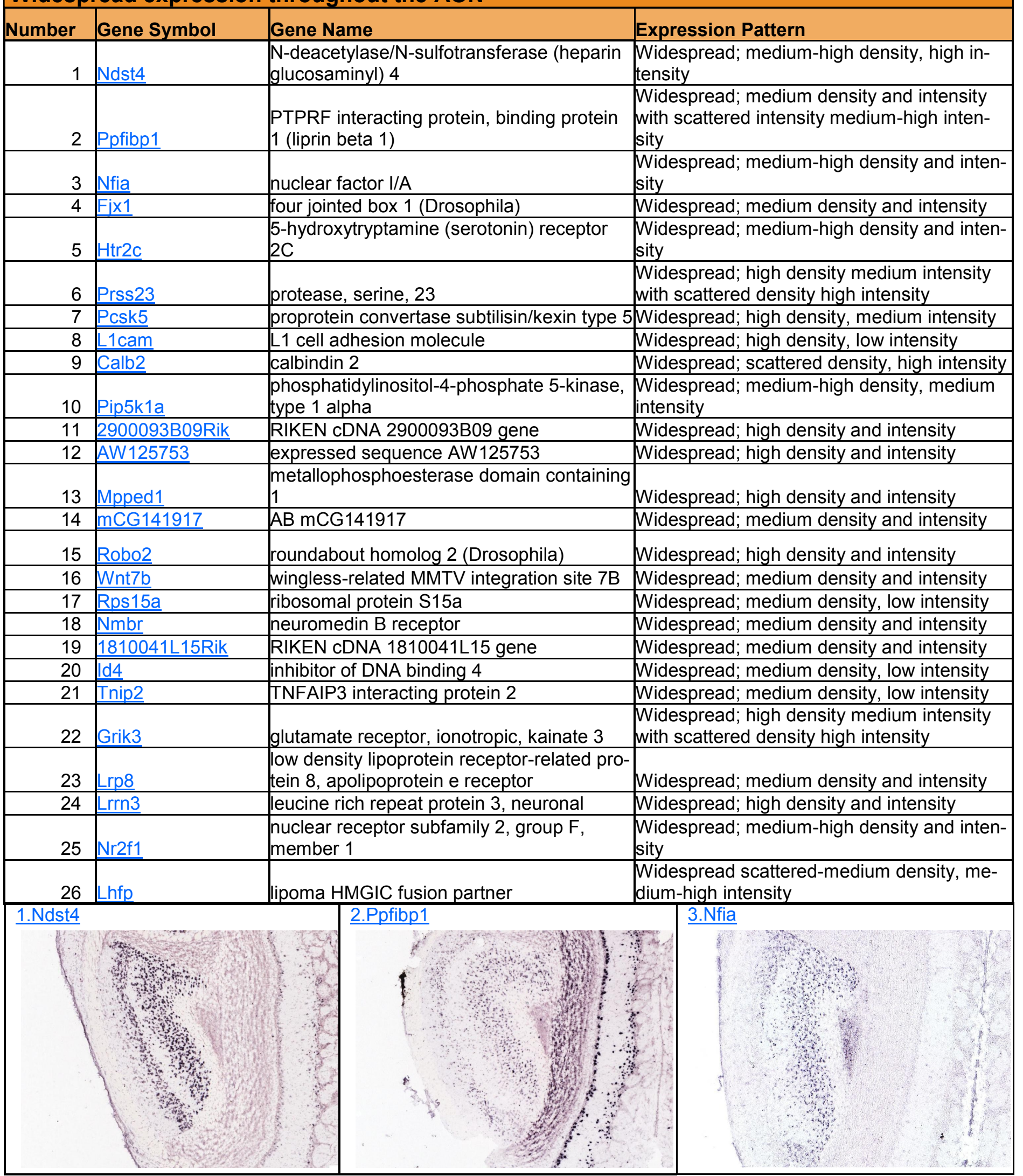




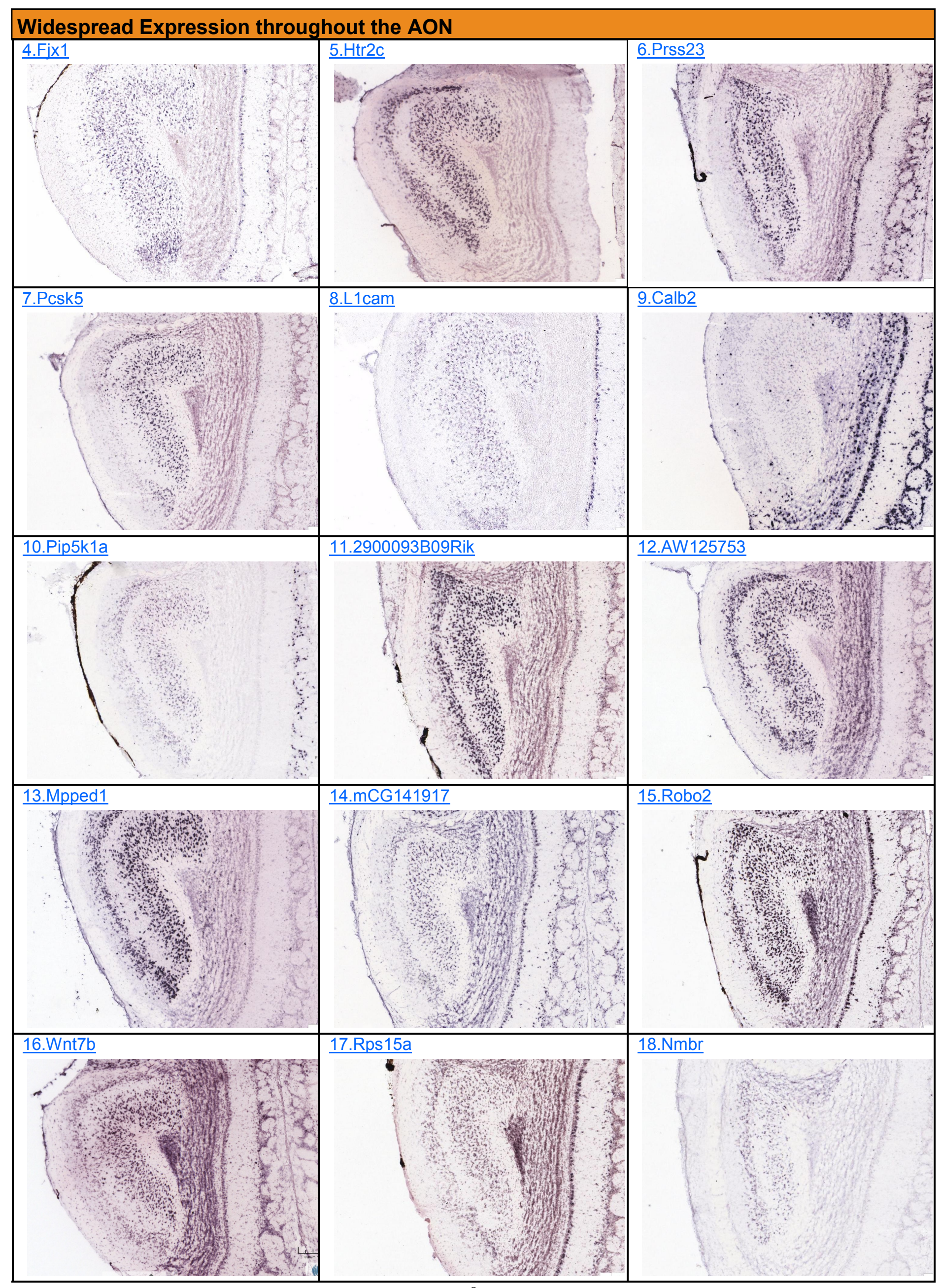




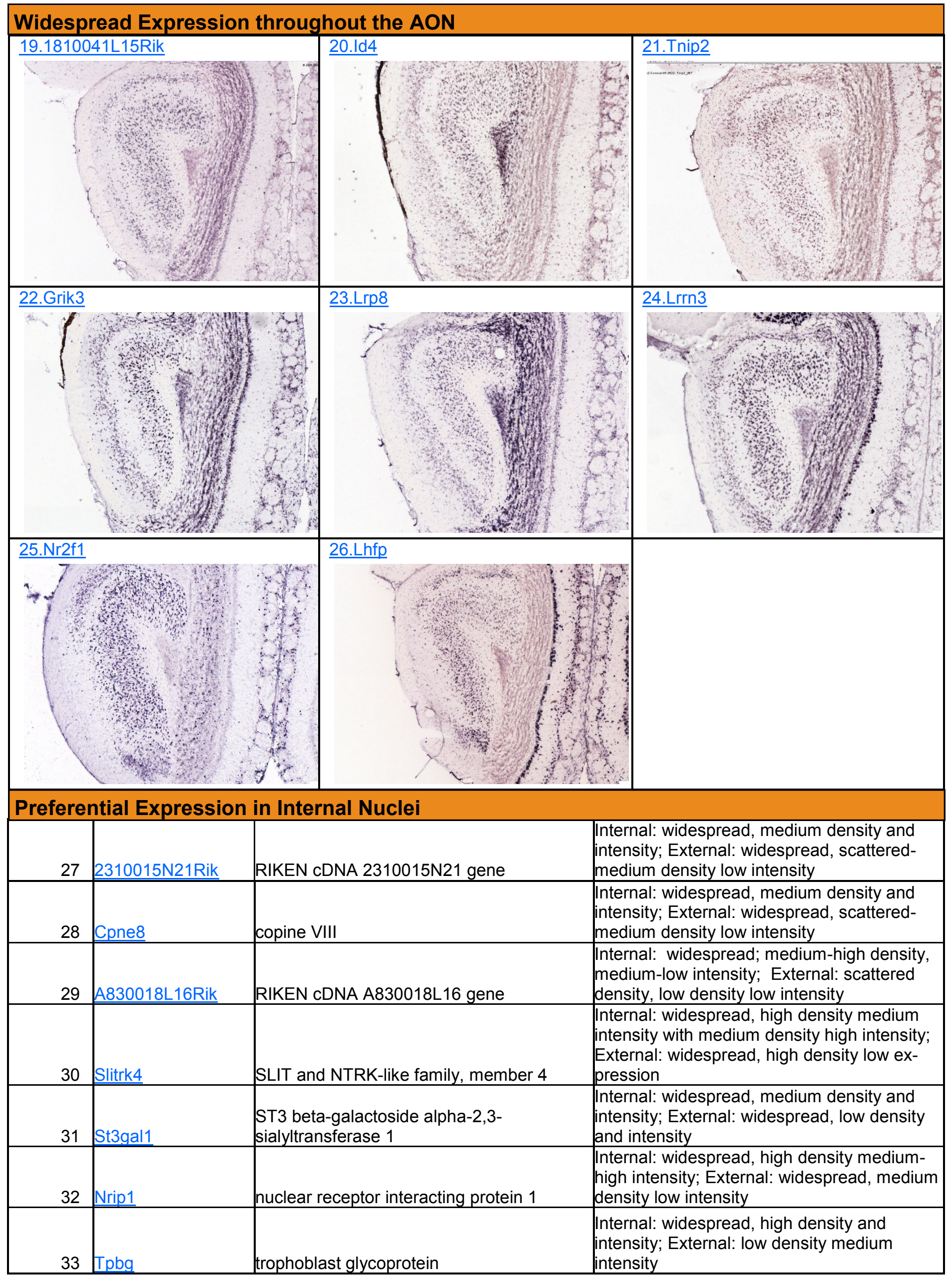




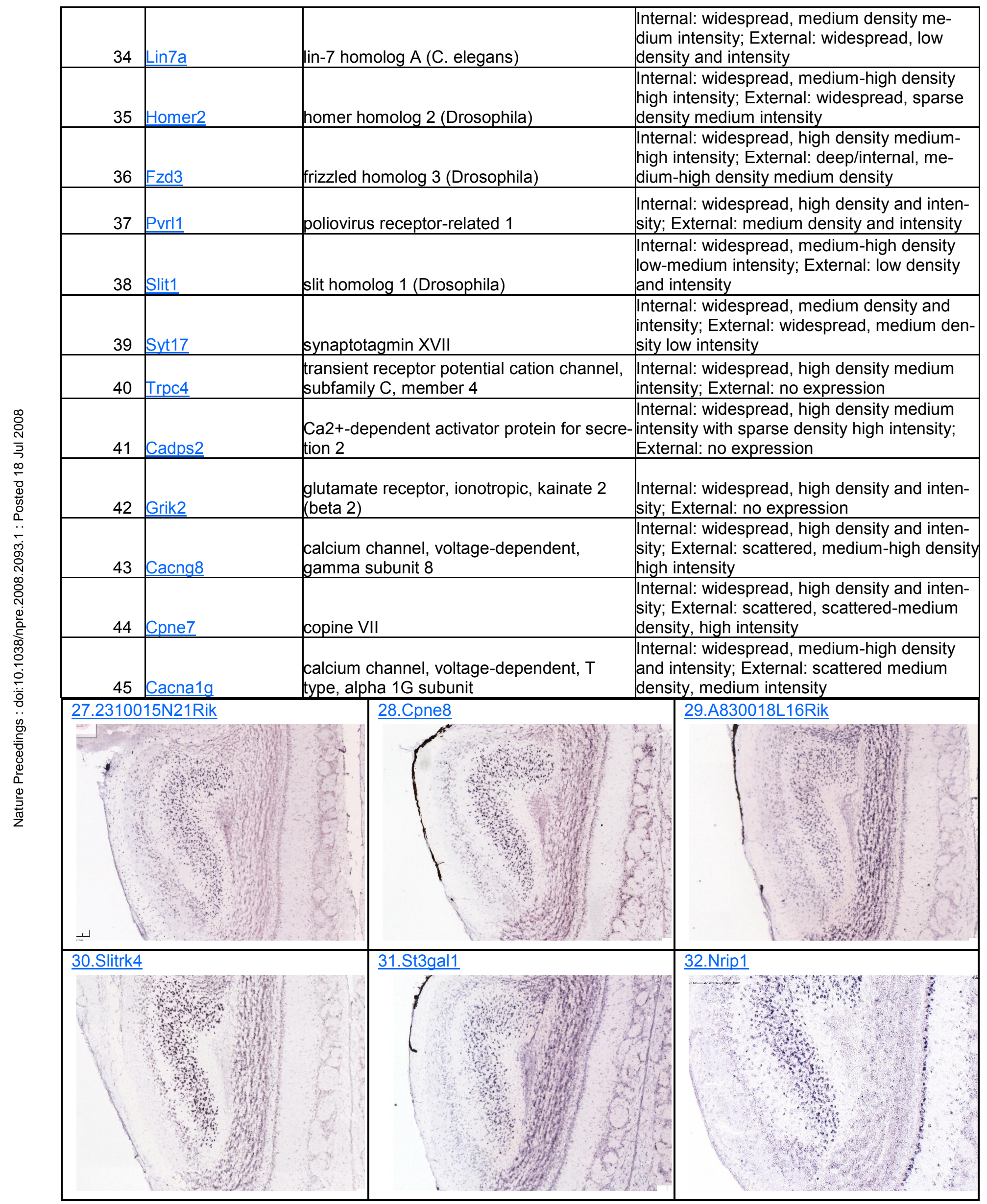




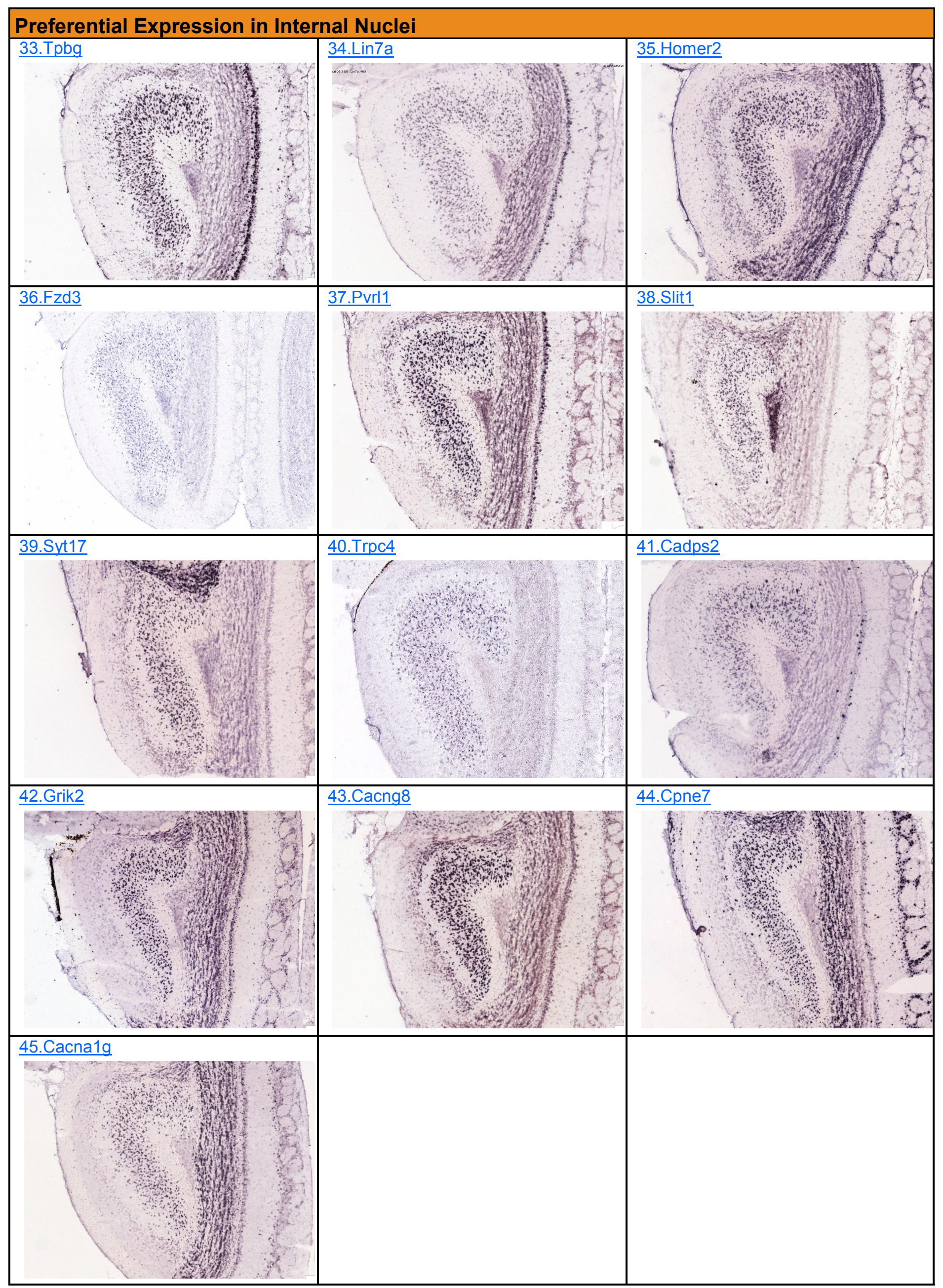




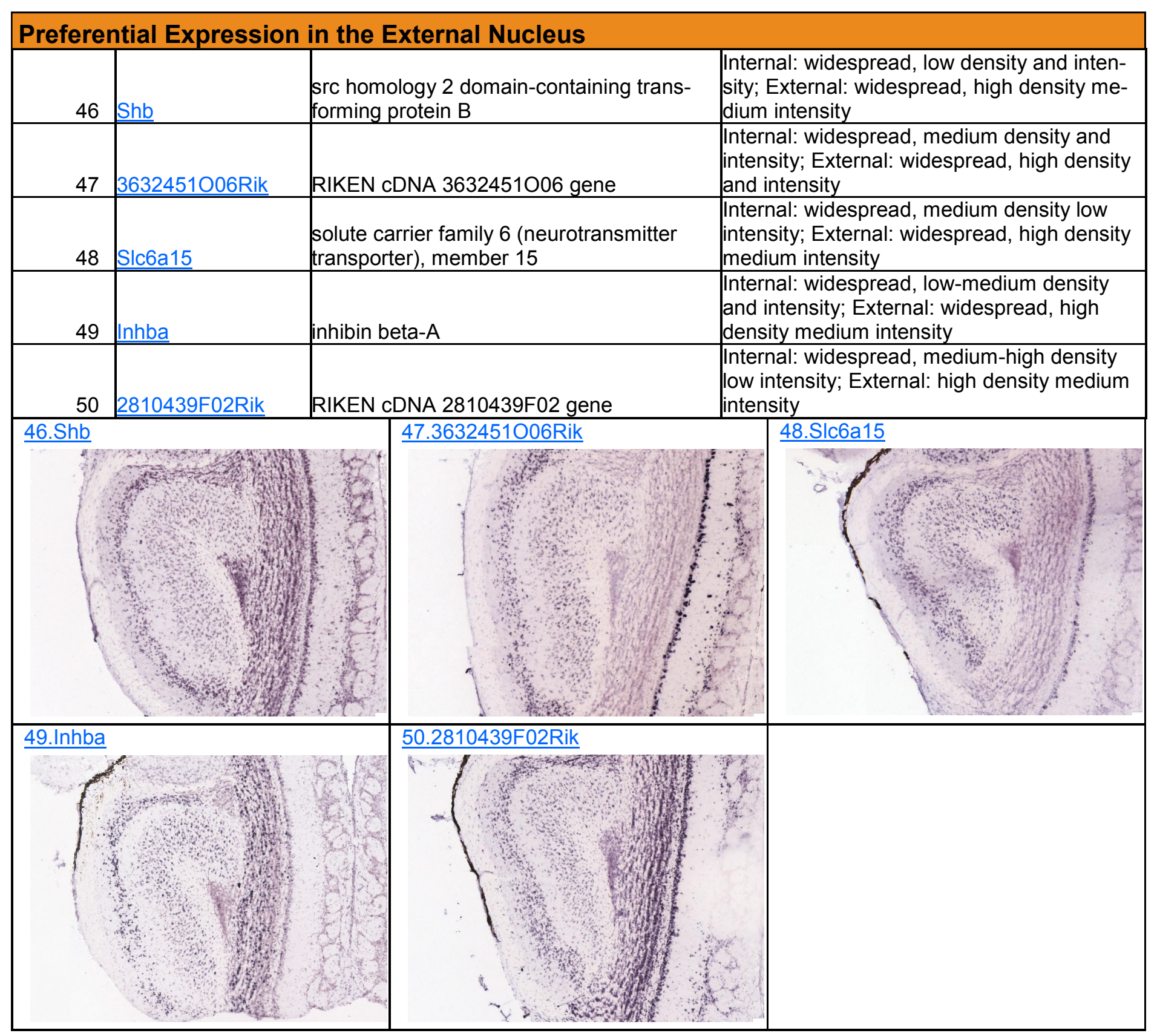


The ABA coronal set contains many genes of known scientific interest as well as genes exhibiting combinatorial or unique expression patterns. A correlation analysis of all available ABA coronal experiments (4376) was performed by comparing the expression value of the $A O N$ to expression values in other regions of the brain. Following image analysis, the data values for each experiment were mapped to a 3-D reference brain at $(200 \mu \mathrm{m})^{3}$ voxel resolution. Then, each voxel was assigned a single expression value based on the product of density and intensity of expression. Values from all 4376 experiments were computed, and the likelihood of coexpression between any two voxels or regions are reported as a Pearson's correlation coefficient.

For the purposes of determining correlated expression between the AON and other brain regions, expression values from all voxels within the AON were aggregated to form a single expression value. Two types of comparisons were then made. First, the aggregate expression values of the AON and those of other anatomically defined regions ( 200 structures) were compared within the 3-D reference brain (structure vs. structure; table below). Second, a color map was then generated to display the correlation between the AON and each of the $\sim 53,000$ voxels of the reference volume (structure vs. voxel; correlation map on the following page).

\section{STRUCTURE vS. STRUCTURE}

The expression value of the AON was compared to expression values for all other defined atlas regions. Degree of correlation is displayed as a comparative fraction, with self-correlation $=1.000$. Correlation between the AON and macro/parent-structures are presented, as well as correlation between the AON and the 25 highest ranking substructures. The most highly correlated macro/parentstructures don't always contain the 25 top most correlated substructures. Columns match the Allen Reference Atlas palette.

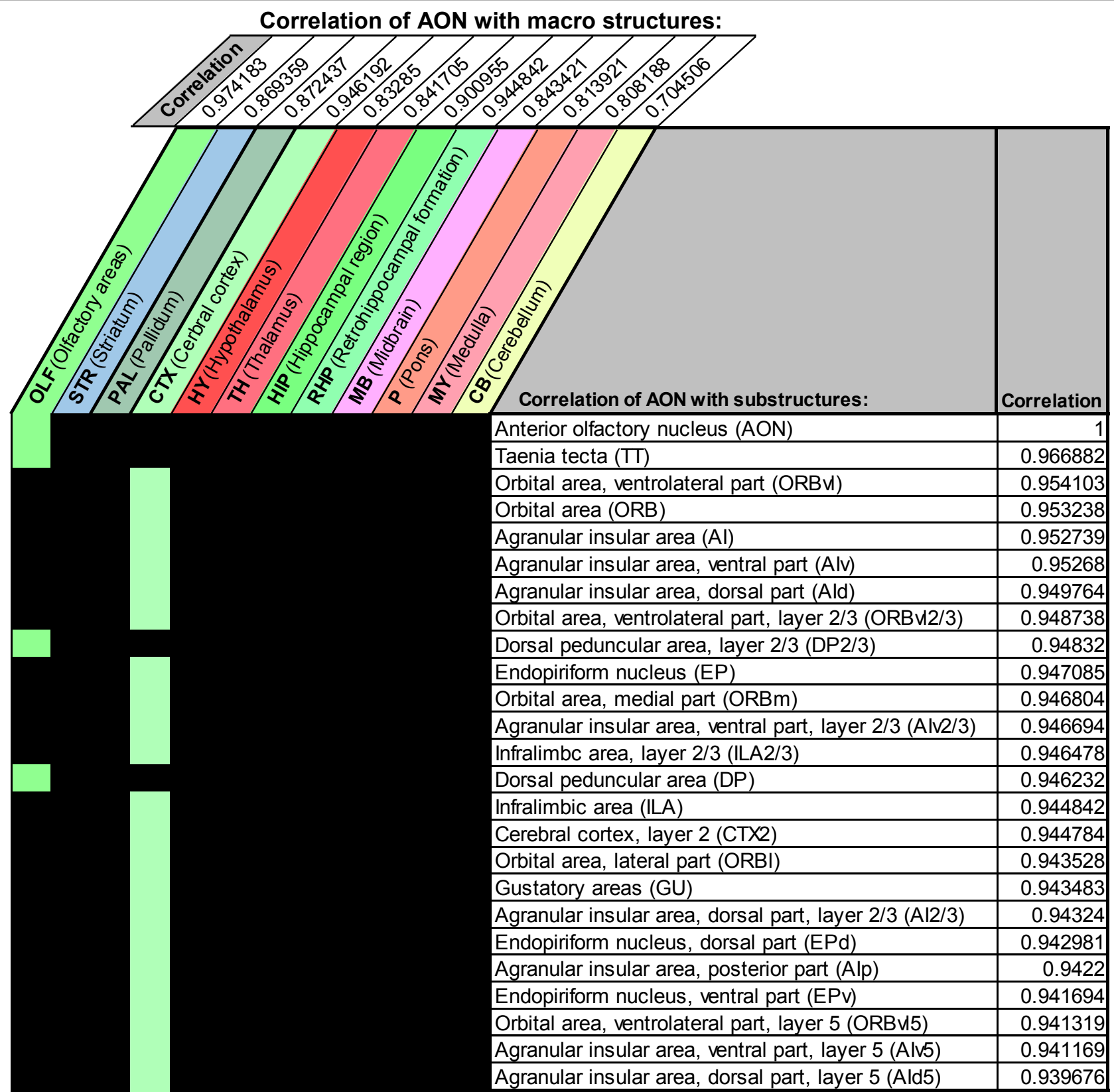




\section{STRUCTURE vS. VOXEL:}

Correlation between the AON and all other $(200 \mu \mathrm{m})^{3}$ voxels in the brain. Degree of correlation assessed for each voxel is provided visually (lower value = the correlation value of the 25th ranked substructure reported on the previous page) using the "jet" color scale at rostrocaudal levels throughout the brain.
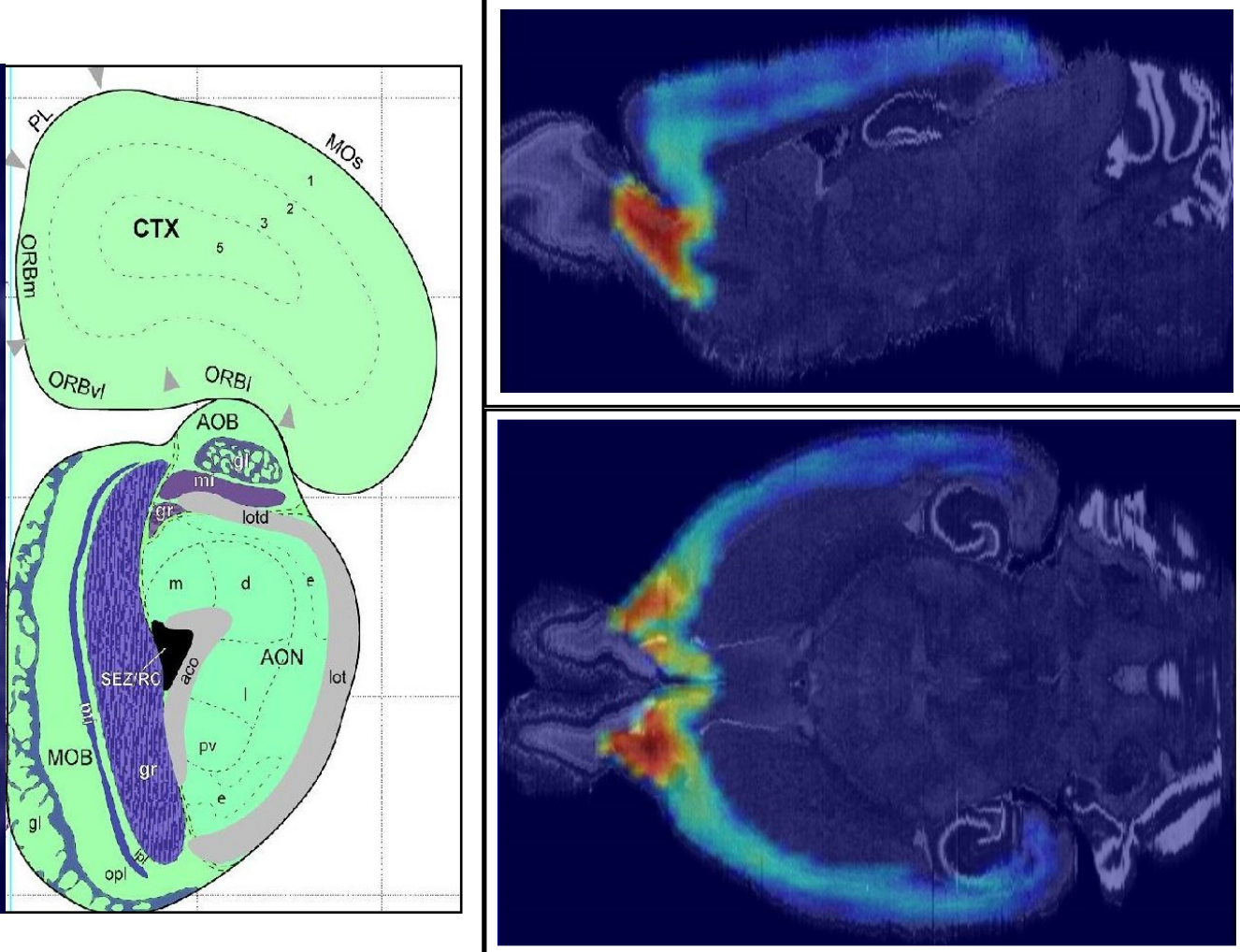

93

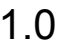

Coronal series through brain:

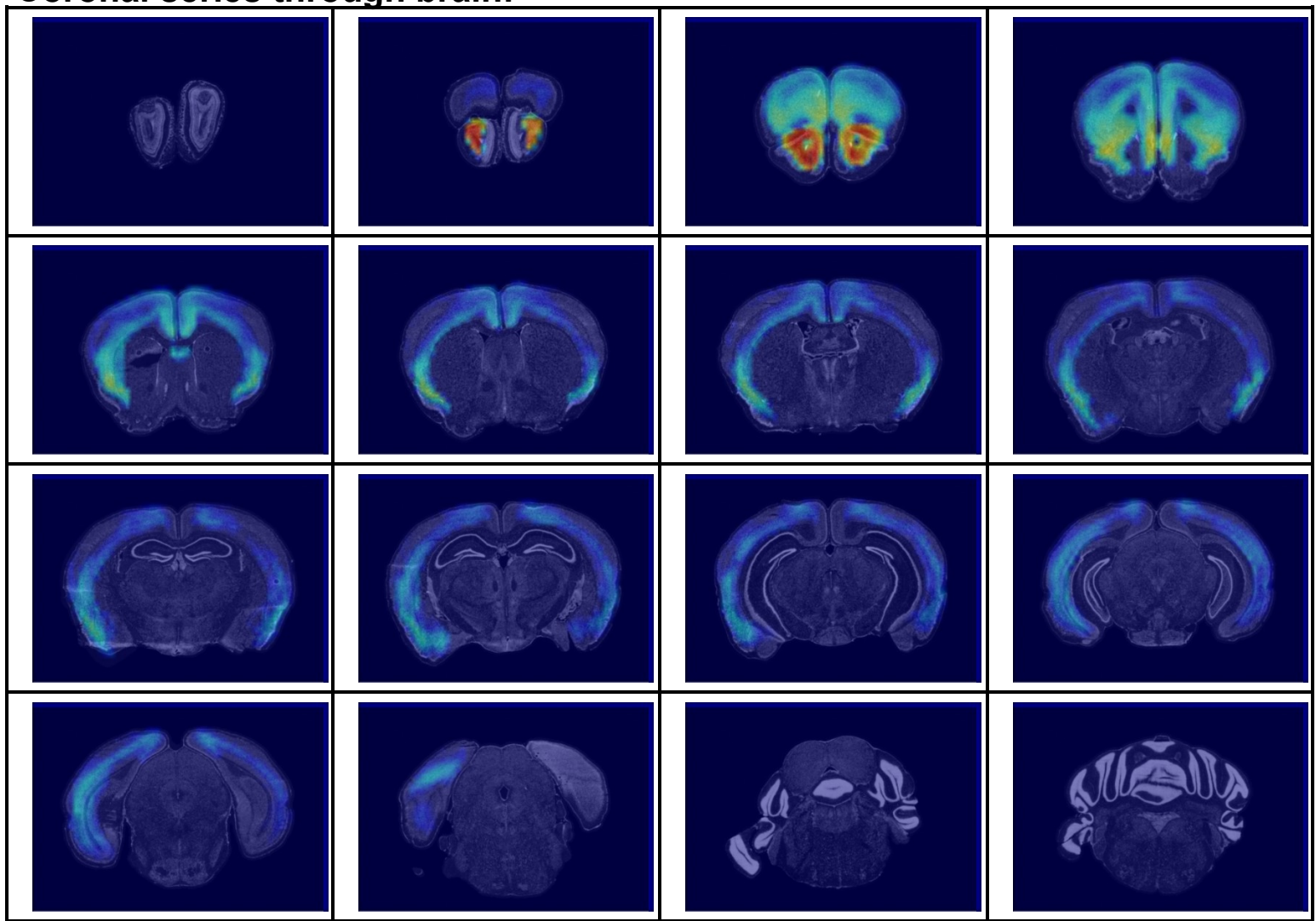




\section{Gene Ontology (GO) Analysis:}

\section{GO TABLE:}

Below is an ontological analysis of the 50 Select Genes, using DAVID Bioinformatics Resources. The functional terms that follow were returned using these constraints:

\begin{tabular}{|l|l|l|}
\hline Category & Definition & Constraints \\
\hline P-value & $\begin{array}{l}\text { Probability that the term is over-represented in this } 50 \\
\text { Select Genes list relative to the mouse genome }\end{array}$ & when p $\leq 0.05$ \\
\hline Gene Count & $\begin{array}{l}\text { The minimum number of genes that must fall into an onto- } \\
\text { logical category to be considered a group }\end{array}$ & 5 genes per term group \\
\hline GO Level & $\begin{array}{l}\text { The level of functional specificity for GO functional cate- } \\
\text { gories: Molecular Function (mf), Biological Process (bp) } \\
\text { and Cellular Components (cc) }\end{array}$ & Level GO_All \\
\hline \# of DAVID IDs & Number of unique DAVID gene IDs from user's input list & 49 DAVID gene IDs / 50 input genes \\
\hline
\end{tabular}

\section{Date of table completion: February 2008}

\begin{tabular}{|l|l|r|r|r|}
\hline GO Category & GO Term & Gene Count & \% of Genes & p-value \\
\hline GOTERM_BP_ALL & neurogenesis & 6 & $12.24 \%$ & 4.12 E-04 \\
\hline GOTERM_BP_ALL & neuron differentiation & 5 & $10.20 \%$ & 0.002440215 \\
\hline GOTERM_BP_ALL & nervous system development & 6 & $12.24 \%$ & 0.006042327 \\
\hline GOTERM_BP_ALL & establishment of localization & 15 & $30.61 \%$ & 0.006877538 \\
\hline GOTERM_BP_ALL & localization & 15 & $30.61 \%$ & 0.007417478 \\
\hline GOTERM_BP_ALL & system development & 6 & $12.24 \%$ & 0.008597943 \\
\hline GOTERM_BP_ALL & metal ion transport & 5 & $10.20 \%$ & 0.011755501 \\
\hline GOTERM_CC_ALL & extracellular region & 14 & $28.57 \%$ & 0.016735434 \\
\hline GOTERM_CC_ALL & extracellular space & 13 & $26.53 \%$ & 0.016878012 \\
\hline GOTERM_BP_ALL & cell differentiation & 7 & $14.29 \%$ & 0.017498944 \\
\hline GOTERM_CC_ALL & plasma membrane & 11 & $22.45 \%$ & 0.020723188 \\
\hline GOTERM_BP_ALL & ion transport & 6 & $12.24 \%$ & 0.025329619 \\
\hline GOTERM_BP_ALL & cation transport & 5 & $10.20 \%$ & 0.028233226 \\
\hline
\end{tabular}

Glynn Dennis Jr., Brad T. Sherman, Douglas A. Hosack, Jun Yang, Michael W. Baseler, H. Clifford Lane, Richard A. Lempicki. "DAVID: Database for Annotation, Visualization, and Integrated Discovery." Genome Biology. 2003 4(5): P3. 


\section{Anatomy}

- The $A O N$ is comprised of external $(A O N e)$, dorsal (AONd), lateral (AONI), medial $(A O N m)$ and posteroventral (AONpv) subnuclei. The cells of the external nucleus are distinctly separate from those of the more difficult to distinguish internal nuclei.

- In the coronal plane, the AON emerges within the posterior portion of the main olfactory bulb (MOB); it remains until the appearance of the nucleus accumbens.

- In the sagittal plane, the AON appears just lateral to the junction of the MOB and the rest of the brain, and remains present until the midline; it is located just caudal to the MOB and rostro-dorsal to the piriform cortex.

\section{Expression Patterns of the $\mathbf{5 0}$ Select Genes}

- The most common expression pattern seen in the AON is widespread expression throughout the entire nucleus.

- A subset of genes show either preferential expression in the external nucleus or preferential expression in the internal nuclei.

\section{Correlation}

- Gene expression in the AON correlated strongly with expression in olfactory and cortical areas situated close to the AON. This included the dorsal peduncular areas and orbital and agranular insular cortices.

- Distinct correlation was also noted throughout the piriform cortex and in layers two/three and five of the cerebral cortex.

- Individual voxel analysis revealed correlation with portions of the amygdala as well.

We encourage you to reply with any comments or questions by email to !Annotation@alleninstitute.org. To further explore the gene expression data and analytical tools referred to in this report, please access our genome-wide data set at brain-map.org.

\section{Other Tools:}

\section{NEUROBLAST:}

Many of the 50 genes listed in this report can be used to explore the NeuroBlast tool. This unique mining tool works seamlessly from within brain-map.org to produce a list of genes that share similar expression patterns to any gene in the coronal data set. Search for and select any gene, then select one of several brain regions from the NeuroBlast drop-tab to explore a ranked list of similarly expressed genes for that region.

To learn more about this function, please refer to the NeuroBlast white paper.

\section{BRAIN EXPLORER:}

To compare gene expression levels across anatomical structures in 3-D detail, download the Brain Explorer desktop application. This program is used to view gene expression in 3-D view (coronal, sagittal, horizontal and everywhere in between) across all brain structures and allows for simultaneous viewing of multiple expression profiles.

The NeuroBlast spatial homology function and an anatomic search tool are also available from within Brain Explorer to allow the user to search for and visualize genes with similar expression patterns. 